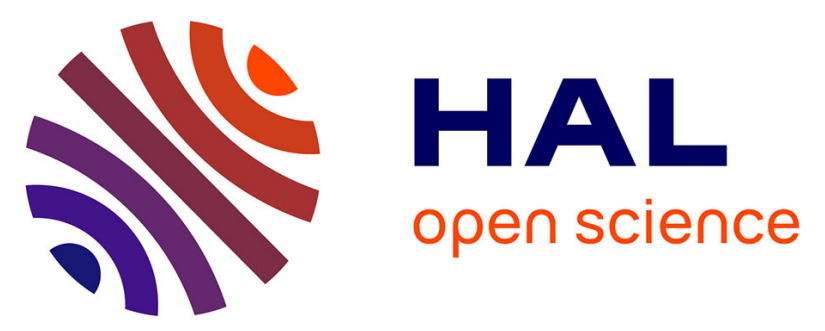

\title{
Comparison of predicted and experimental subcellular localization of two putative rat steroid dehydrogenases from the short-chain dehydrogenase/reductase protein superfamily
}

\author{
Brigitte Keller, Marc Meier, Jerzy Adamski
}

\section{To cite this version:}

Brigitte Keller, Marc Meier, Jerzy Adamski. Comparison of predicted and experimental subcellular localization of two putative rat steroid dehydrogenases from the short-chain dehydrogenase/reductase protein superfamily. Molecular and Cellular Endocrinology, 2009, 301 (1-2), pp.43. 10.1016/j.mce.2008.07.022 . hal-00532058

\section{HAL Id: hal-00532058 \\ https://hal.science/hal-00532058}

Submitted on 4 Nov 2010

HAL is a multi-disciplinary open access archive for the deposit and dissemination of scientific research documents, whether they are published or not. The documents may come from teaching and research institutions in France or abroad, or from public or private research centers.
L'archive ouverte pluridisciplinaire HAL, est destinée au dépôt et à la diffusion de documents scientifiques de niveau recherche, publiés ou non, émanant des établissements d'enseignement et de recherche français ou étrangers, des laboratoires publics ou privés. 


\section{Accepted Manuscript}

Title: Comparison of predicted and experimental subcellular localization of two putative rat steroid dehydrogenases from the short-chain dehydrogenase/reductase protein superfamily

Authors: Brigitte Keller, Marc Meier, Jerzy Adamski

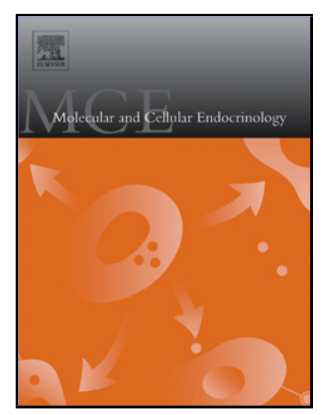

PII:

S0303-7207(08)00330-4

DOI: doi:10.1016/j.mce.2008.07.022

Reference: MCE 6932

To appear in: Molecular and Cellular Endocrinology

Received date: 3-7-2008

Revised date: 24-7-2008

Accepted date: 24-7-2008

Please cite this article as: Keller, B., Meier, M., Adamski, J., Comparison of predicted and experimental subcellular localization of two putative rat steroid dehydrogenases from the short-chain dehydrogenase/reductase protein superfamily, Molecular and Cellular Endocrinology (2007), doi:10.1016/j.mce.2008.07.022

This is a PDF file of an unedited manuscript that has been accepted for publication. As a service to our customers we are providing this early version of the manuscript. The manuscript will undergo copyediting, typesetting, and review of the resulting proof before it is published in its final form. Please note that during the production process errors may be discovered which could affect the content, and all legal disclaimers that apply to the journal pertain. 
Comparison of predicted and experimental subcellular localization of two putative rat steroid dehydrogenases from the short-chain dehydrogenase/reductase protein superfamily

Brigitte Keller ${ }^{1 \#}$, Marc Meier ${ }^{1 \#}$, and Jerzy Adamski ${ }^{1,2 *}$

${ }^{1}$ Helmholtz Zentrum München, German Research Center for Environmental Health, Institute for Experimental Genetics, Genome Analysis Center, Ingolstaedter Landstr. 1, 85764 Neuherberg, Germany

${ }^{2}$ Lehrstuhl für Experimentelle Genetik, Technische Universität München, Germany

\# contributed equally to the work

*To whom correspondence should be addressed: adamski@helmholtz-muenchen.de, Tel. +49893187 3155, Fax: +498931873225 


\begin{abstract}
In the characterization of newly identified proteins, subcellular localization studies can provide important hints to the proteins' metabolic functions. Depending on the biochemical task of an enzyme, certain subcellular environmental conditions as $\mathrm{pH}$ or availability of cofactors and substrates have to be fulfilled. Consequently, misdirected proteins often cannot conduct the proper chemical reaction.

This study is aimed at detecting differences in bioinformatic and wet lab experiments and presenting ways for reliable analysis of subcellular localization. On a set of ten enzymes from the short-chain dehydrogenase/reductase (SDR) superfamily, we have performed predictions and experimental analyses of subcellular localization. Exemplarily, we show the localization studies on rat short-chain dehydrogenases/reductases dhrs $7 \mathrm{~b}$ and dhrs8. We demonstrate in particular that all of the prediction algorithms tested failed to assign the SDR enzymes to an experimentally verified subcellular compartment.
\end{abstract}

\title{
KEYWORDS
}

Subcellular localization, short-chain dehydrogenases/reductases, SDRs, dhrs7b, dhrs8 


\section{Introduction}

For several frequent human diseases like metabolic syndrome, obesity, diabetes or epithelial cancers the molecular mechanisms underlying the pathogenesis involve large protein families sharing some structural motifs like that of the aldo-keto reductases or the short-chain dehydrogenase/reductases (SDR) (Oppermann et al., 2003) which comprise many uncharacterized members (Keller et al., 2006). In the characterization of novel proteins, subcellular localization studies can provide important hints to their metabolic functions. Depending on the biochemical task of an enzyme, certain subcellular environmental conditions as $\mathrm{pH}$ or availability of substrates and cofactors have to be fulfilled. Consequently, misdirected proteins often cannot conduct the proper chemical reaction. The significance of appropriate environment becomes apparent considering as example many hydroxysteroid dehydrogenases which have shown ability to catalyze different directions of reactions in vivo or under defined conditions in vitro (Khan et al., 2004).

For preliminary bioinformatic analysis several servers running different algorithms are publicly available. The challenge in the SDR proteins is that their high level of structure conservation (and hydrophobicity patterns) is unparalleled by amino acid sequence dissimilarity. We compared five servers for a set of ten so far uncharacterized SDR enzymes. Remarkably, the results differ widely or are nonconclusive depending on the algorithms used. Therefore, we verified the issue by recombinant expression of the respective proteins in living cells. To avoid potential masking of the targeting signals we expressed the proteins as $\mathrm{N}$ - or C-terminally tagged recombinant proteins, respectively. By counterstaining of several compartments and visualization by fluorescent microscopy, we were then able to precisely define the subcellular localization.

This study is aimed at highlighting differences in bioinformatics and wet lab experiments and presenting ways for reliable analysis of subcellular localization. For ten SDRs, we have conducted predictions and experimental analyses of subcellular localization. Exemplarily for 
these studies, localization of previously non studied rat short-chain dehydrogenases/ reductases dhrs $7 \mathrm{~b}$ and dhrs8 will be shown.

\section{Material and Methods}

Bioinformatic prediction of subcellular localization

Five servers were employed for the analysis of subcellular localization. These servers were:

pSORTII (http://psort.ims.u-tokyo.ac.jp/form2.html), pTarget

(http://bioinformatics.albany.edu/ ptarget/), SubLoc1.1

(http://www.bioinfo.tsinghua.edu.cn/SubLoc/eu_predict.htm), TargetP1.1

(http://www.cbs.dtu.dk/services/TargetP/), LOCtree

(http://cubic.bioc.columbia.edu/services/loctree). For references, see respective web sites and references therein.

\section{Cloning of rat dhrs $7 b$ and rat dhrs 8}

For cloning, the CDS were amplified by PCR from IMAGE clones ordered from RZPD (dhrs7b: IMAGp998M1012446Q3, dhrs8: IMAGp958L083175Q2). Dhrs7b was cloned into pEGFP-C2 (Becton\&Dickinson, Karlsruhe, Germany) and pcDNA4 myc-his Version B (Invitrogen, Karlsruhe, Germany) in frame by use of EcoRI and BamHI restriction sites or EcoRI and SacII restriction sites, respectively (PCR primers. pEGFP-C2: forward 5'- TAT AGA ATT CAT GCT CAA GGA GAG GGC CAT GGA C-3', reverse 5'- TTA AGG ATC CTC AGG AGT TCT TGG ATT TTC GCT C-3'; pcDNA4 myc-his: forward 5'- TAT AGG ATC CAT GCT CAA GGA GAG GGC CAT GGA C-3', reverse 5'-TTA ACC GCG GGG AGT TCT TGG ATT TTC GCT CTT T-3'). Dhrs8 was cloned into pEGFP-C2 and pAcGFP1-N1 (both Becton\&Dickinson, Karlsruhe, Germany) in frame by use of EcoRI and BamHI restriction sites (PCR primers forward (both vectors) 5'- TAT AGA ATT CAT GAA GTA CCT TCT TGA CCT GAT C-3', reverse (pEGFP-C2) 5'- TTA AGG ATC CTC ATT 
TAT CTT TGT AGC CAA CAA C-3', reverse (pAcGFP1-N1) 5'- TTA AGA ATT CTT TAT CTT TGT AGC CAA CAA CAG C-3'). Inserts were verified by didesoxy sequence analysis using vector specific primers. For transfection, DNA was isolated using the PureYield Midi Kit (Promega, Mannheim, Germany) according to the manufacturer's instructions.

\section{Cell culture and transfection}

HeLa cells were grown under humidified standard conditions $\left(37^{\circ} \mathrm{C}, 5 \% \mathrm{CO} 2\right)$ in $\mathrm{High}$ Glucose Dulbecco’s Modified Eagle Medium (Invitrogen, Karlsruhe, Germany) supplemented with 10\% FBS (Biochrom AG, Berlin, Germany), 2 mM LGlutamaxI (Invitrogen), and 100 $\mathrm{U} / \mathrm{ml}$ penicillin/100 $\mu \mathrm{g} / \mathrm{ml}$ streptomycin (also Invitrogen). For transfection, FuGENE6 transfection reagent (Roche Biosciences, Mannheim, Germany) was used according to the manufacturer's instructions.

\section{Immunocytochemistry and staining of subcellular compartments}

For analysis of subcellular localization, HeLa cells were seeded onto cover slides. $24 \mathrm{~h}$ after seeding, cells were transfected and grown for further $24 \mathrm{~h}$. Fixation was done by $3.7 \%$ formaldehyde in PBS, permeabilization with $0.1 \%$ Triton-X100 in PBS.

For staining of the endoplasmic reticulum (living cell staining), ER-Tracker (Invitrogen) was used. Alternatively, staining was conducted by co-transfection of rabbit N-FLAG-Sarcolipin (Odermatt et al., 1998). The FLAG tag was detected by a respective antibody (anti FLAG, rabbit, Sigma, Taufkirchen, Germany), and as a secondary antibody, Alexa Fluor 568 goat anti-rabbit IgG (Invitrogen) was used. For immunochemical detection of the myc-Tag, MycTag (9B11) Mouse mAb (Cell Signaling, NEB, Frankfurt a.M., Germany) was used as primary antibody, the secondary antibody was Alexa Fluor 488 goat anti-mouse IgG (Invitrogen). Counterstaining of the nuclei was conducted with DAPI, and of f-actin by use of 
AlexaFluor568-coupled phalloidin (both Invitrogen) according to the manufacturer's protocol. After fixation and staining, cover slides were mounted onto microscope slides using Vectashield (Vector Laboratories, Burlingame, CA, USA). Subcellular localization was analyzed by fluorescence microscopy on a Zeiss Axiophot (Carl Zeiss, Oberkochen, Germany) microscope using a 40x oil immersion objective and the ISIS (MetaSystems, Altlussheim, Germany) software.

\section{Results}

Bioinformatic prediction of subcellular localization

For the prediction of subcellular localization, we used five publicly available servers (pSORTII, pTarget, SubLoc, TargetP, and LOCtree) employing different algorithms. As shown in Tables 1 and 2, the predictions differ widely for the given proteins.

\section{Experimental analysis of subcellular localization}

Due to limited reliability of bioinformatic prediction methods, recombinant expression of the respective proteins and subsequent analysis by immunochemistry was indispensable. To avoid potential masking of the targeting signals we expressed the proteins from pEGFP-C2 and pAcGFP1-N1, resulting in $\mathrm{N}$ - or C-terminally GFP-tagged recombinant proteins. Alternatively, for rat dhrs7b, a C-terminal myc-his tag was added by the use of pcDNA4 mycHis Version B and the resulting fusion protein detected by antibody staining of the myc-tag. By fluorescent microscopy and counterstaining of several subcellular organelles, we were then able to precisely define the subcellular localization.

As shown in fig. 1 (panel 2), dhrs7b distribution in the cells co-localizes with rabbit sarcolipin. Rabbit sarcolipin is known to localize to the endoplasmic reticulum (Odermatt et 
al., 1998). We therefore conclude that rat dhrs7b is localized in the endoplasmic reticulum. Fig. 2 shows the intracellular localization of rat dhrs8. By overlay with the ER tracker we can also show endoplasmic reticulum localization of this protein.

\section{Problems caused by GFP overexpression in cell staining}

After overexpression of GFP-tagged recombinant proteins, predominantly after expression from pAcGFP1-N1, we partially observed inconclusive results. This fact is illustrated in fig. 3. The protein under investigation (in this case mDhrs4, Keller et al., in preparation) shows cytosolic distribution. Image A (expression from pcDNA4, detection of the myc-tag) clearly confirms this observation. However, after expression from pAcGFP1-N1 a particulate distribution can only be supposed (images B to D). The blobs detected are inclusions formed. They are often seen after expression from the pAcGFP1-N1 vector but not in other vectors. Only in picture B, cytosolic distribution can be envisioned.

\section{Discussion}

On ten SDRs (for two proteins, experimental data are shown here - the other eight will be discussed elsewhere), predictions on the subcellular localization were conducted by use of the five different public servers pSORTII, pTarget, SubLoc, TargetP, and LOCtree. Although the aforementioned bioinformatical tools are only a small selection out of currently more than 20 publicly available servers (Schneider and Fechner, 2004), the provided and here shown results can be esteemed as well representative. On the other hand, our design of protein expression in eukaryotic cells while using alternating either $\mathrm{N}$ - or $\mathrm{C}$-terminal tagging prevented targeting signal masking. Avoiding this interference provided us with several sound experimental observations on subcellular localisation.

Overall, the prediction of subcellular localization is not reliable. This was seen for more than one protein under investigation. For one examined enzyme, all predictions were different, in 
most cases at least two servers agreed on the same localization. Concerning three proteins all predictions proved wrong, although all servers predicted the same localization. In summary, different servers tend to deliver different predictions, depending on the algorithms they use. While TargetP, for instance, relies exclusively on the prediction of $\mathrm{N}$-terminal sequences (Emanuelsson et al., 2000), SubLoc judges the overall amino acid composition (Hua and Sun, 2001). Table 3 summarizes the correct and false predictions, evaluated on the basis of wet lab study results.

Looking at the findings in detail, no clear preference can be deduced for certain subcellular compartments to be accurately predicted. Experimental data shows that out of ten enzymes investigated, two are localized in the mitochondria. Both were properly predicted by pTarget, the latter also by SubLoc1.1. From two cytoplasmic proteins, one was incorrectly predicted by all servers. The other one, in contrast, was correctly foretold by the pSORTII, pTarget and LOCtree servers. For the proteins localized in the endoplasmic reticulum, this observation does not change.

Predictions on the subcellular localization can not considered to be reliable at least concerning the enzymes under investigation in this study. It is unlikely that non-SDR type enzymes use different localization signals from the SDRs under investigation here. If conducted at all, these predictions should therefore be handled with great care. From the servers used, pSORTII and pTarget showed the best performance - with $40 \%$ correct predictions. This rather poor performance is due to several facts (for a review, see Schneider and Fechner, 2004): there are unconventional and, in addition, most likely many yet unknown targeting signals. Furthermore, co-translational pathways for protein import into organelles have been described (Crowley and Payye, 1998) but can so far not be recognized by the prediction algorithms. This fact is also true for proteins, reaching several cellular compartments due to specific protein interactions (backpacking). It additionally should be taken into consideration however that protein localization does not have to be static but can change. For example, 
hWWOX (human WW domain containing oxidoreductase) has been shown to localize to mitochondria. Upon activation, it is translocated to the nucleus (Chang, 2002). It is therefore possible that supposedly incorrect predictions are, in fact, alternative localizations under different circumstances.

However, the method of bioinformatic prediction, applied in this investigation, is commonly used and should therefore provide greater reliability. We suggest introducing several secondary features to the prediction algorithms to improve their fidelity. They may include for mitochondrial proteins the analysis for existence of protease cleavage site for the hydrophobic mitochondrial leading peptide, and for endoplasmic reticulum the number of potential intramolecular disulphide bonds. 


\section{Acknowledgements}

We thank Dr. A. Odermatt (University Basel, Switzerland) for his kind gift, the N-FLAG rabbit sarcolipin plasmid, used for ER counterstaining. 


\section{Literature}

Chang, N.S., 2002. A potential role of p53 and WOX1 in mitochondrial apoptosis (review). Int. J. Mol. Med. 9 (1), 19-24.

Crowley, K.S., Payye, R.M., 1998. Ribosome binding to mitochondria is regulated by GTP and the transit peptide. J. Biol. Chem. 273, 17278-17285.

Emanuelsson, O., Nielssen, H., Brunak, S., von Heijne, G., 2000. Predicting subcellular Localization of proteins based on their N-terminal amino acid sequence. J. Mol. Biol. 300, $1005-1016$.

Hua, S., Sun, Z., 2001. Support vector machine approach for protein subcellular localization prediction. Bioinformatics. 17 (8), 721-728.

Keller, B., Volkmann, A., Wilckens, T., Moeller, G., Adamski, J., 2006. Bioinformatic identification and characterization of new members of short-chain dehydrogenase/reductase superfamily. Mol. Cell. Endocrinol. 248, 56-60.

Khan, N., Sharma, K.K., Andersson, S., Auchus, R.J., 2004. Human 17beta-hydroxysteroid dehydrogenases types 1,2 , and 3 catalyze bi-directional equilibrium reactions, rather than unidirectional metabolism, in HEK-293 cells. Arch. Biochem. Biophys. 429, 50-59.

Odermatt, A., Becker, S., Khanna, V.K., Kurzydlowski, K., Leisner, E., Pette, D., MacLennan, D.H., 1998. Sarcolipin Regulates the Activity of SERCA1, the Fast-twitch 
Skeletal Muscle Sarcoplasmic Reticulum Ca2+-ATPase. J. Biol. Chem. 273 (20), 1236012369.

Oppermann, U., Filling, C., Hult, M., Shafqat, N., Wu, X., Lindh, M., Shafqat, J., Nordling, E., Kallberg, Y., Persson, B., and Jornvall, H., 2003. Short-chain dehydrogenases/reductases (SDR): the 2002 update. Chem Biol Interact. 143-144, 247-253.

Schneider, G., Fechner, U., 2004. Advances in the predictions of protein targeting signals. Proteomics. 4, 1571-1580. 


\section{Legends to figures}

Figure 1. Subcellular localization of rat dhrs $7 b$.

Rat dhrs 7b is located in the ER. Panel 1: A ER staining, B GFP-dhrs7b, C overlay of A and B. Rat dhrs7b was expressed from pEGFP-C2 resulting in a N-terminally EGFP-tagged recombinant protein of cytosolic distribution. Panel 2: A nuclear counterstaining, B detection of C-terminally myc-tagged rat dhrs $7 b, C$ ER counterstaining by use of rabbit N-FLAG sarcolipin, D overlay of A to C. Co-localization of sarcolipin and dhrs $7 \mathrm{~b}$ is especially apparent in upper left corner (orange arrowhead). Purple box outlines background staining from myc-tag antibody.

Figure 2. Subcellular localization of rat dhrs8.

Rat dhrs8 is located in the ER. In both panels, picture A shows ER staining, B GFP-dhrs8 and dhrs8-GFP respectively and $\mathrm{C}$ overlay of $\mathrm{A}$ and $\mathrm{B}$. Panel 1: expression from pEGFP-C2, Panel 2: expression from pAcGFP1-N1.

Figure 3. Problems encountered in determination of subcellular localization of a cytosolic protein from pAcGFP1-N1.

A: Expression from pcDNA4 myc-his Version B. B to D: Inconclusive localization after expression from pAcGFP1-N1. Blobs are inclusions formed. This is often seen after expression from this vector. All cells were stained with DAPI (nuclear counterstaining) and phalloidin (f-actin). 
Table 1. Predictions on the subcellular localization of rat dhrs $7 \mathrm{~b}$

\section{Server rat dhrs7b}

pSORTII cytoplasmic 39.1\%, mitochondrial $30.4 \%$, vacuolar $8.7 \%$, extracellular (incl. cell wall) $8.7 \%$, endoplasmic reticulum $8.7 \%$, nuclear $4.3 \%$

pTarget cytoplasmic $75.1 \%$

SubLoc1.1 mitochondrial 50.0\%

TargetP1.1 mitochondrial $42.9 \%$, secretory $4.1 \%$, other $45.6 \%$

LocTree cytoplasmic -3 
Table 2. Predictions on the subcellular localization of rat dhrs 8

\section{Server rat dhrs8}

pSORTII cytoplasmic 47.8\%, mitochondrial 21.7\%, extracellular (incl. cell wall) $17.4 \%$, vacuolar $4.3 \%$, endoplasmic reticulum $4.3 \%$, nuclear $4.3 \%$

pTarget endoplasmic reticulum $87.6 \%$

SubLoc1.1 cytoplasmic $84.0 \%$

TargetP1.1 mitochondrial $1.7 \%$, secretory $96.8 \%$, other $6.3 \%$

LocTree mitochondrial - 5 
Table 3. Evaluation of subcellular localization predictions on a set of ten SDRs

\begin{tabular}{lccc}
\hline Server & $\boldsymbol{\Sigma}$ & correct & false \\
pSORTII & 10 & 4 & 6 \\
pTarget & 10 & 4 & 6 \\
SubLoc & 10 & 1 & 9 \\
TargetP & 10 & 0 & 10 \\
LocTree & 10 & 1 & 9 \\
\hline
\end{tabular}



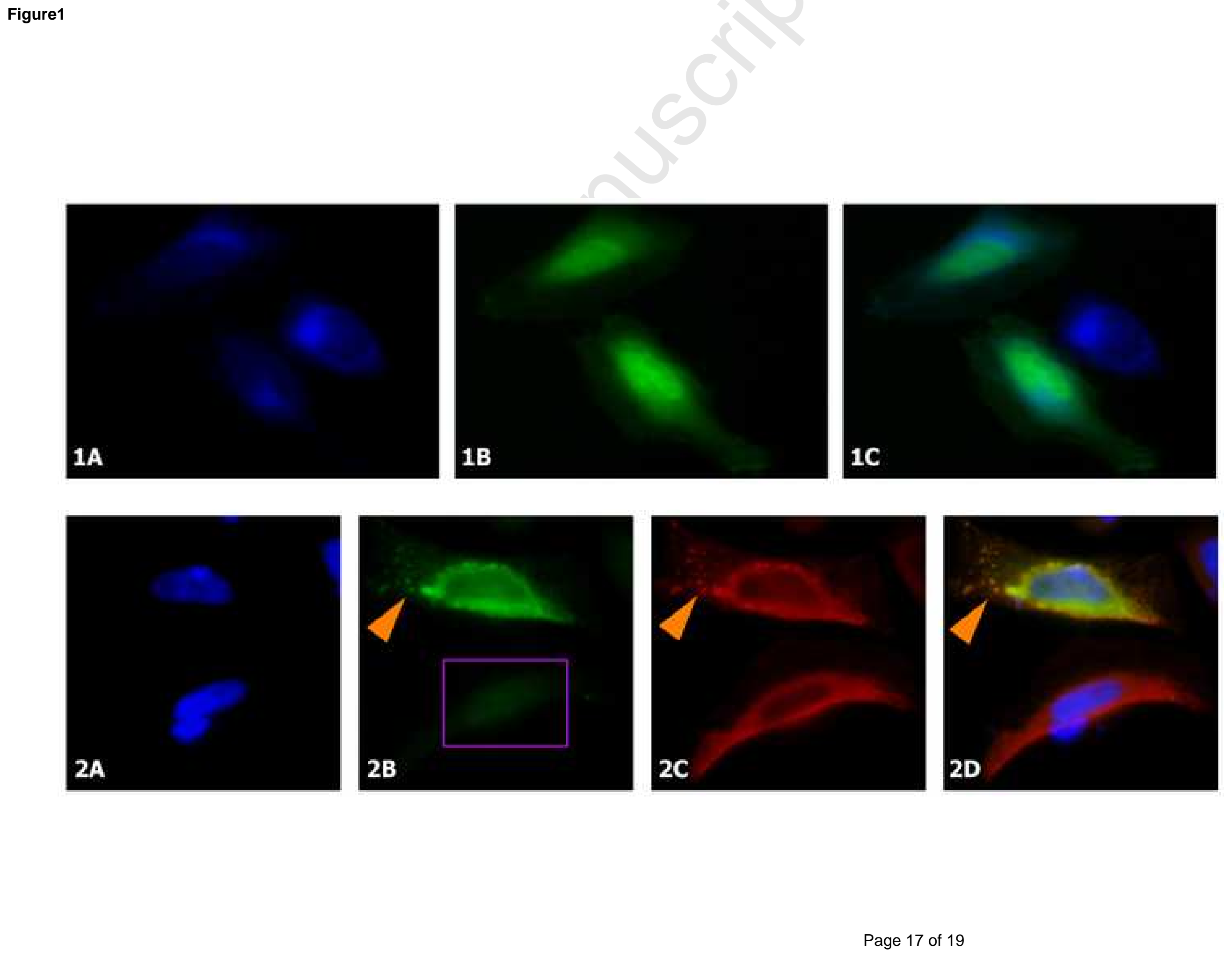

Page 17 of 19

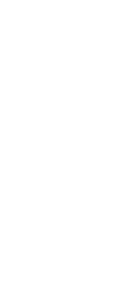

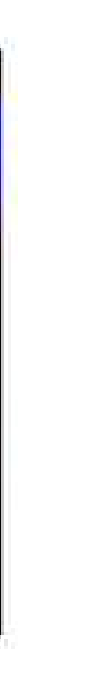
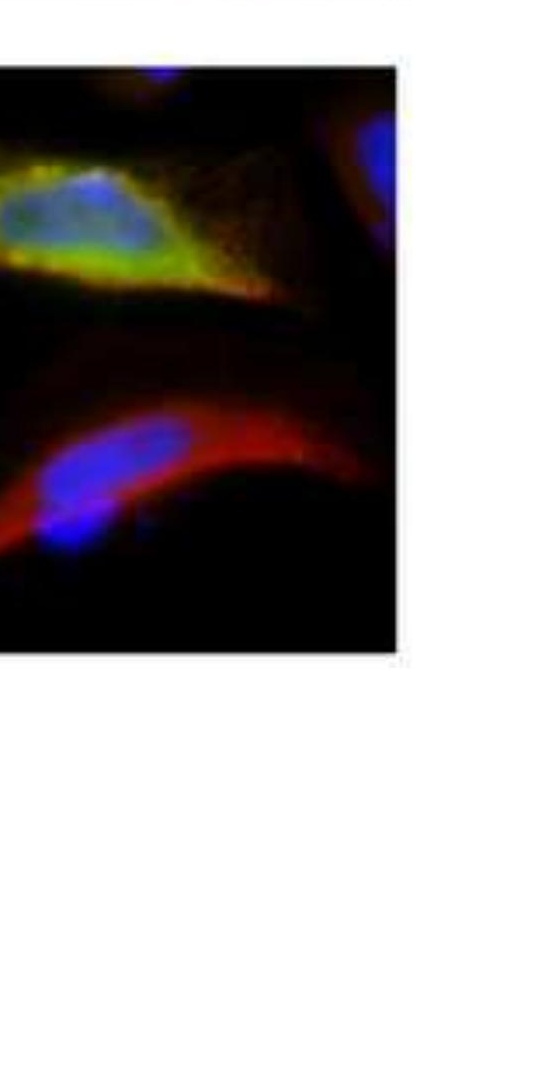

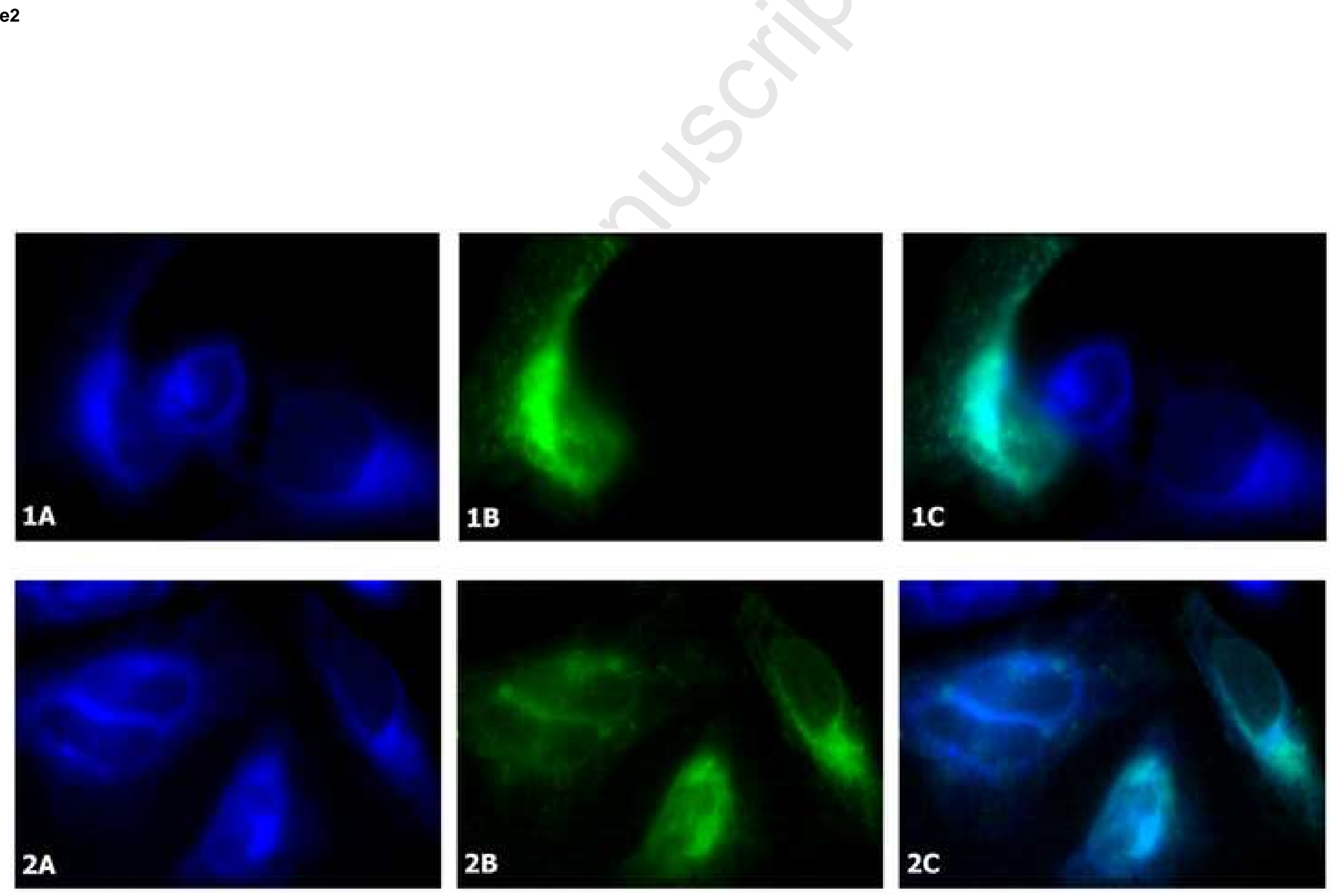

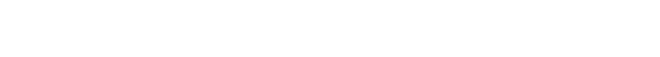

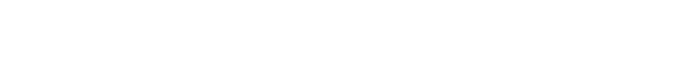

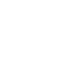


